\title{
Carmustine Wafers (Gliadel) Plus Concomitant Temozolomide Therapy After Resection of Malignant Astrocytoma: Growing Evidence for Safety and Efficacy
}

\author{
Matthew J. McGirt, MD and Henry Brem, MD \\ Johns Hopkins University School of Medicine, Baltimore, MD
}

Menei and colleagues have performed a thorough analysis in their retrospective cohort study from 26 centers in France. They report the overall survival and complication rate associated with the adjuvant use of implantable biodegradable carmustine wafers (Gliadel; MGI Pharma, Bloomington, $\mathrm{MN}$; now Eisai Inc.) for the treatment of primary and recurrent malignant astrocytoma (MA). The authors also investigate the independent association of morbidity and overall survival with combination therapy of temozolomide (TMZ, Stupp protocol) and Gliadel implantation after surgical resection. ${ }^{1,2}$

In their series of 83 primary and 80 recurrent MA treated with surgical resection plus Gliadel implantation, the authors report a median survival of 7 months after revision resection of recurrent $\mathrm{MA}$ and 17 months after primary resection of MA. For the 43 patients receiving both Gliadel and Stupp protocol after primary resection of MA, a median survival of 608 days (approximately 19.9 months) was observed. Consistent with previous studies, factors independently associated with survival were fractionated radiotherapy, Karnofsky performance score, and extent of resection. The proportions of these prognostic variables were comparable to prior Gliadel series. This series of Gliadel implantation used a variety of adjuvant radiation and chemotherapy regimens. Hence, the overall survival was greater than those previously reported in randomized trails of Gliadel for primary MA. ${ }^{3,4}$ Although the retrospective nature of this study precludes direct comparison, variations in patient characteristics, tumor grades, extent of surgical resection, and the addition of adjuvant TMZ likely

(C) Society of Surgical Oncology 2010

Published Online: 27 April 2010

H. Brem, MD

e-mail: hbrem@jhmi.edu underlies the prolonged survival reported in this Gliadel series.

Until the advent of Gliadel wafers, radiation remained the only adjuvant treatment to surgery that prolonged survival for patients with MA. Gliadel wafers, which provide local delivery of the chemotherapeutic polymer 1,3-bis(2chloroethyl)-1-nitrosourea (BCNU), has consistently been reported to extend median survival by 2 to 4 months for patients with newly diagnosed and recurrent MA. ${ }^{2-6}$ More recently, temozolomide (Temodar, Schering Corp.) became only the second adjuvant therapy in addition to surgery and radiation to prolong survival for MA, increasing median survival by 2.5 months when administered concomitantly with postoperative radiotherapy. 1,7

Combining immediately delivered local chemotherapy via Gliadel and more delayed systemic chemotherapy via the Stupp protocol offers many theoretical advantages that may underlie the prolonged survival observed here. First, systemic TMZ is most effective in regions of tumor that are most vascular, whereas local delivery of carmustine allows direct access of the chemotherapeutic agent independent of vasculature. In exposed resection cavities that are relatively avascular after surgical hemostasis, Gliadel allows treatment of residual tumor cells that present a theoretical obstacle to systemic TMZ. Second, postoperative radiotherapy plus concomitant and adjuvant TMZ does not deliver chemotherapeutic agent to the debulked tumor during the 3-week period between surgery and radiotherapy. Gliadel wafer delivery of local BCNU offers a theoretical bridge of this nontherapeutic period. Hence, the combination of Gliadel wafer implantation with concomitant and adjuvant TMZ treatment theoretically allows continuous adjuvant therapy for up to 9 months, beginning immediately after tumor resection. The 19.9-month median survival reported here with TMZ plus Gliadel supports this approach and is 
consistent with the prospective phase II study by La Rocca et al., which reported an 18.6-month median survival after surgical resection with TMZ plus Gliadel. ${ }^{8}$

In our initial experience with the combination of surgery plus Gliadel and TMZ (Stupp) for primary MA, we observed a median survival of 20.7 months and 2-year survival of $36 \% .^{9}$ Patient age, extent of resection, and Karnofsky performance score status in our experience are comparable to the series reported here. The favorable overall survival reported by Menei and colleagues is likely due to the addition of TMZ to their Gliadel practice, a multimodal treatment approach that has consistently yielded favorable results. ${ }^{8-10}$

The morbidity associated with Gliadel wafers has been well reported in the literature. Menei and colleagues report adverse events in $44.6 \%$ of patients receiving Gliadel implantation with primary surgical resection and in $43.8 \%$ of patients with revision resection. Although these rates of adverse events are higher than that observed in our experience, their reported complications that are more directly associated with the surgical site are comparable to previous reports. $^{2-6,9,11}$ After primary resection, the authors report seizures in $4.8 \%$ of patients, intracranial hypertension in $9.6 \%$, septic abscess in $6 \%$, and wound healing difficulty in $2.4 \%$ of patients receiving Gliadel. Revision surgery was associated with a similar complication incidence, with the exception of greater seizure $(10 \%)$ and wound healing difficulty (7.4\%). As highlighted by the authors, these complications were similar to those reported in the phase III trial by Westphal et al. and highlight the importance of perioperative corticosteroids and antiepileptics in patients receiving Gliadel. ${ }^{4}$ Furthermore, the combination of the Stupp TMZ protocol and Gliadel implantation was not associated with increased morbidity of any type in this series. ${ }^{1-6,8}$

In our 10-year experience with Gliadel, we have implanted Carmustine wafers in nearly 300 patients undergoing resection of MA. ${ }^{11}$ This series, which remains the largest reported cohort of Gliadel implantation, demonstrated surgical site infection in $2.8 \%$ (most of which were revision resection for recurrence), wound healing abnormality in $0.7 \%$, cerebrospinal fluid leak in $2.8 \%$, malignant intracranial hypertension in $2.1 \%$, and perioperative seizures in $14.6 \%$ of patients. Among our 725 patients who received surgical resection without Gliadel during the same time period, the incidence of these perioperative complications was nearly identical. Furthermore, in our initial experience with the combination of surgery plus Gliadel and concomitant TMZ for primary MA, we have observed a complication rate similar to that of Gliadel alone or postoperative TMZ alone. ${ }^{9}$ After Gliadel plus concomitant TMZ therapy, surgical site infection occurred in $3 \%$ of patients, seizures in $6 \%$ of patients, and malignant intracranial hypertension in $3 \%$ of patients. TMZ-induced myelosuppression was the most clinically important complication observed, occurring in $21 \%$ of patients receiving Gliadel and TMZ via the Stupp protocol. It has been our experience that with watertight dural closure, several weeks' use of corticosteroids and antiepileptics, and avoidance of Gliadel implantation adjacent to an open ventricle or an exposed cerebral artery, perioperative morbidity can be expected to be unaffected by Gliadel use alone or in combination with TMZ.

The retrospective cohort study by Menei and colleagues adds to the growing literature that Gliadel and concomitant TMZ (Stupp protocol) can be administered together safely after resection of MA. A prospective randomized trial is warranted to determine the relative efficacy of Gliadel plus concomitant TMZ versus TMZ alone after resection of MA. This past decade has seen a doubling of the median survival for MA from 9 months to $>18$ months. Newer innovative methods of direct delivery of therapeutic agents are being developed and warrant continued assessment in clinical trials. ${ }^{12,13}$

\section{REFERENCES}

1. Stupp R, Mason WP, van den Bent MJ, et al. Radiotherapy plus concomitant and adjuvant temozolomide for glioblastoma. $N$ Engl J Med. 2005;352:987-96.

2. Brem H, Piantadosi S, Burger PC, et al. Placebo-controlled trial of safety and efficacy of intraoperative controlled delivery by biodegradable polymers of chemotherapy for recurrent gliomas. The Polymer-brain Tumor Treatment Group. Lancet. 1995;345: 1008-12.

3. Valtonen S, Timonen U, Toivanen $\mathrm{P}$, et al. Interstitial chemotherapy with carmustine-loaded polymers for high-grade gliomas: a randomized double-blind study. Neurosurgery. 1997;41:44-8.

4. Westphal M, Hilt DC, Bortey E, et al. A phase 3 trial of local chemotherapy with biodegradable carmustine (BCNU) wafers (Gliadel wafers) in patients with primary malignant glioma. Neurooncology. 2003;5:79-88.

5. Brem H, Ewend MG, Piantadosi S, et al. The safety of interstitial chemotherapy with BCNU-loaded polymer followed by radiation therapy in the treatment of newly diagnosed malignant gliomas: phase I trial. J Neurooncol. 1995;26:111-23.

6. Westphal M, Ram Z, Riddle V, Hilt D, Bortey E. Gliadel wafer in initial surgery for malignant glioma: long-term follow-up of a multicenter controlled trial. Acta Neurochir (Wien). 2006;148: 269-75.

7. Gallia GL, Brem S, Brem H. Local treatment of malignant brain tumors using implantable chemotherapeutic polymers. J Natl Compr Canc Netw. 2005;3:721-8.

8. La Rocca RV, Villanueva WG, Vitaz TW, et al. A phase II study of radiation with concomitant and then sequential temozolomide (TMZ) in patients with newly diagnosed supratentorial highgrade malignant glioma who have undergone surgery with carmustine (BCNU) wafer insertion. Neurooncology. 2006;8:391500 .

9. McGirt MJ, Than KD, Weingart JD, et al. Gliadel (BCNU) wafer plus concomitant temozolomide therapy after primary resection of glioblastoma multiforme. J Neurosurg. 2009;110:583-8. 
10. Rich JN, et al. Overall survival of primary glioblastoma (GBM) patients receiving carmustine (BCNU) wafers followed by radiation (RT) and concurrent temozolomide (TMZ) plus rotational multi-agent chemotherapy. J Clin Oncol. 2007;25:2070.

11. Attenello FJ, Mukherjee D, Datoo G, et al. Use of Gliadel (BCNU) wafer in the surgical treatment of malignant glioma: a 10-year institutional experience. Ann Surg Oncol. 2008;15: 2887-93.
12. Menei P, Capelle L, Guyotat J, et al. Local and sustained delivery of 5-fluorouracil from biodegradable microspheres for the radiosensitization of malignant glioma: a randomized phase II trial. Neurosurgery. 2005;56:242-8.

13. Quinn JA, Jiang SX, Carter J, et al. Phase II trial of Gliadel plus O6-benzylguanine in adults with recurrent glioblastoma multiforme. Clin Cancer Res. 2009;15:1064-8. 